Annals of Pure and Applied Mathematics

Vol. 16, No. 2, 2018, 413-418

ISSN: 2279-087X (P), 2279-0888(online)

Published on 13 March 2018

Annals of

www.researchmathsci.org

DOI: http://dx.doi.org/10.22457/apam.v16n2a18

\title{
Generalized Maximal Closed Sets in Topological Space
}

\author{
Suwarnlatha N. Banasode ${ }^{1}$ and Mandakini A.Desurkar ${ }^{2}$ \\ ${ }^{1}$ Department of Mathematics, K.L.E. Society's, R.L.Science Institute \\ Belgaum - 590001, India \\ E-mail :suwarn_nam@yahoo.co.in \\ ${ }^{2}$ Department of Mathematics, KLS Gogte Institute of Technology \\ Belagavi-590008, India. E-mail :mdesurkar9@gmail.com \\ ${ }^{2}$ Corresponding author
}

Received 19 February 2018; accepted 9 March 2018

Abstract. In this paper, we introduce and study generalized maximal closed sets in topological space and obtain some of their properties. A subset $\mathrm{A}$ of $\mathrm{X}$ is said to be generalized maximal closed (briefly $\mathrm{g}-\mathrm{m}_{\mathrm{a}}$ closed) set in a topological space $(\mathrm{X}, \tau)$, if $\mathrm{cl}$ (A) $\subseteq \mathrm{U}$ whenever $\mathrm{A} \subseteq \mathrm{U}$ and $\mathrm{U}$ is maximal open set in $\mathrm{X}$.

Keywords: Minimal closed, generalized minimal closed, maximal open set, $\omega$-closed set

AMS Mathematics Subject Classification (2010): 54A05, 54B05

\section{Introduction and preliminaries}

The notion of closed set is fundamental in the study of topological spaces. In 1970, Levine [1] introduced the concept of generalized closed sets in topological spaces by comparing the closure of a subset with its open supersets. Further the study of g-closed sets was continued by Dunham and Levine [1]. Maximal open sets and Minimal open sets were studied and introduced by Nakaoka and Oda [3,4,5]. Benchalli, Banasode and Siddapur introduced and studied generalized minimal closed sets in topological spaces [2]. Further Banasode and Desurkar introduced and studied generalized minimal closed sets in bitopological spaces [7].

Throughout this paper $(X, \tau)$ represents a nonempty topological space on which no separation axioms are assumed unless otherwise explicitly stated.

For a subset $\mathrm{A}$ of a topological space $(\mathrm{X}, \tau) \mathrm{cl}(\mathrm{A})$, int $(\mathrm{A})$ and $\mathrm{A}^{\mathrm{c}}$ denote the closure of $\mathrm{A}$, the interior of $\mathrm{A}$ and the complement of $\mathrm{A}$ in $(\mathrm{X}, \tau)$ respectively. Let us recall the following definitions, which are useful in the sequel.

Here $\operatorname{int}^{*}(\mathrm{~A})$ denotes the interior of generalized open set $\mathrm{A}$ and $\mathrm{cl}^{*}(\mathrm{~A})$ denotes the closure of generalized closed set A.

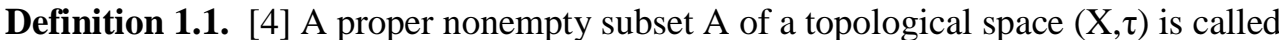
(i) a minimal open (resp. minimal closed) set if any open (resp. closed) subset of $\mathrm{X}$ which is contained in $\mathrm{A}$, is either $\mathrm{A}$ or $\phi$

(ii) a maximal open (resp. maximal closed) set if any open (resp. closed) set which contains $\mathrm{A}$, is either $\mathrm{A}$ or $\mathrm{X}$. 
Suwarnlatha N. Banasode and Mandakini A.Desurkar

Definition 1.2. [1] A subset A of a topological space $(X, \tau)$ is called

(i) a generalized closed (briefly g-closed) set if cl (A) $\subseteq \mathrm{U}$ whenever $\mathrm{A} \subseteq \mathrm{U}$ and $\mathrm{U}$ is an open set in X.

(ii) a generalized open (briefly g-open ) set iff $\mathrm{A}^{\mathrm{c}}$ is a g-closed set.

(iii) a $\omega$-closed set if $\mathrm{cl}(\mathrm{A}) \subseteq \mathrm{U}$ whenever $\mathrm{A} \subseteq \mathrm{U}$ and $\mathrm{U}$ is a semi open set in $(\mathrm{X}, \tau)$.

(iv) an $\omega$-open set iff $\mathrm{A}^{\mathrm{c}}$ is a $\omega$-closed set.

(iii) [2] a generalized minimal closed (briefly g-mi closed) set if cl (A) $\subseteq \mathrm{U}$ whenever

$\mathrm{A} \subseteq \mathrm{U}$ and $\mathrm{U}$ is a minimal open set in $\mathrm{X}$.

\section{Generalized maximal closed sets}

Definition 2.1. A subset $A$ of a topological space $(X, \tau)$ is said to be generalized maximal closed (briefly g- $\mathrm{m}_{\mathrm{a}}$ closed ) set if $\mathrm{cl}(\mathrm{A}) \subseteq \mathrm{U}$ whenever $\mathrm{A} \subseteq \mathrm{U}$ and $\mathrm{U}$ is a maximal open set in $\mathrm{X}$.

Theorem 2.2. Every $g-m_{a}$ closed sets are $\omega$ - closed set.

Proof: Let $\mathrm{V}$ be a $\mathrm{g}-\mathrm{m}_{\mathrm{a}}$ closed set . By definition $2.1 \mathrm{cl}(\mathrm{V}) \subseteq \mathrm{U}$ whenever $\mathrm{V} \subseteq \mathrm{U}$ and $\mathrm{U}$ is maximal open set. We know that every maximal open set is open and also every open sets are semi-open sets. This implies $\mathrm{U}$ is a semi-open set. Therefore $\operatorname{cl}(\mathrm{V}) \subseteq \mathrm{U}$, whenever $\mathrm{V} \subseteq \mathrm{U}$ and $\mathrm{U}$ is semi-open set. Hence $\mathrm{V}$ is $\omega$ - closed set.

Remark 2.3. The converse of the above theorem is not true.

Example 2.4. Let $X=\{a b c\}$ with $\tau=\{X, \phi,\{a\},\{c\},\{a c\},\{a b\}\}$

$\mathrm{m}_{\mathrm{a}}$ - open sets $=\{\mathrm{a} c\},\{\mathrm{a} b\}$

open sets $=\mathrm{X}, \phi,\{\mathrm{a}\},\{\mathrm{c}\},\{\mathrm{a} \mathrm{c}\},\{\mathrm{a} b\}$

closed sets $=\mathrm{X}, \phi,\{\mathrm{b}\},\{\mathrm{c}\},\{\mathrm{a} b\},\{\mathrm{b} \mathrm{c}\}$

$\mathrm{g}-\mathrm{m}_{\mathrm{a}}$ closed sets $=\phi,\{\mathrm{b}\},\{\mathrm{c}\},\{\mathrm{a} b\}$

$\omega$ - closed sets $=\phi,\{b\},\{c\},\{a b\},\{b c\}, X$

$\{\mathrm{b}$ c $\}$ is $\omega$ - closed set but not $\mathrm{g}-\mathrm{m}_{\mathrm{a}}$ closed set.

Theorem 2.5. Every $g-m_{a}$ closed set is g-closed set.

Proof: Let V be g-ma closed set. By Definition $2.1 \mathrm{cl}(\mathrm{V}) \subseteq \mathrm{U}$. Whenever $\mathrm{V} \subseteq \mathrm{U} \& \mathrm{U}$ is maximal open set. We know that every maximal open set is open. This implies $U$ is an open set. Therefore $\mathrm{cl}(\mathrm{V}) \subseteq \mathrm{U}$, whenever $\mathrm{V} \subseteq \mathrm{U} \& \mathrm{U}$ is an open set . Therefore $\mathrm{V}$ is $\mathrm{g}$ closed set.

Remark 2.6. The converse of the above theorem is not true.

Example 2.7. Let $X=\{a \mathrm{~b} c\}$ with $\tau=\{\mathrm{X}, \phi,\{\mathrm{a}\}\}$

Closed set $=\mathrm{X}, \phi,\{\mathrm{b} \mathrm{c}\} ;$ Maximal open $=\{\mathrm{a}\}$

$\mathrm{g}-\mathrm{m}_{\mathrm{a}}$ closed set $=\phi$

g-closed set $=\phi,\{b\},\{c\},\{a b\},\{b c\},\{a \mathrm{c}\}, X$.

Therefore $\{b\}$ is a g-closed set but not $g-m_{a}$ closed set. 
Generalized Maximal Closed Sets in Topological Space

Theorem 2.7. Every $g-m_{i}$ closed set is $g-m_{a}$ closed set.

Proof: Let $\mathrm{V}$ be a $\mathrm{g}-\mathrm{m}_{\mathrm{i}}$ closed set. By definition [2] $\mathrm{cl}(\mathrm{V}) \subseteq \mathrm{U}$. Whenever $\mathrm{V} \subseteq \mathrm{U}$ and $\mathrm{U}$ is minimal open set. Let $A$ be a maximal open set then by [6] either $U \subseteq A$ or it is disconnected. Therefore $\mathrm{U} \subseteq \mathrm{A}$. Thus $\operatorname{cl}(\mathrm{A}) \subseteq \mathrm{U} \subseteq \mathrm{A}$ where $\mathrm{A}$ is a maximal open set. Thus $\mathrm{A}$ is $\mathrm{g}-\mathrm{m}_{\mathrm{a}}$ closed set.

Remark 2.8. The converse of the above theorem is not true.

Example 2.9. Let $X=\{a, b, c, d\}$ and $\tau=\{X, \phi,\{a\},\{a, b\},\{c, d\}\{a, c, d\}\}$

$\mathrm{g}-\mathrm{m}_{\mathrm{i}}$ closed sets : $\phi,\{\mathrm{c}\},\{\mathrm{d}\},\{\mathrm{c}, \mathrm{d}\}$

g-m $\mathrm{m}_{\mathrm{a}}$ closed sets: $\phi,\{\mathrm{b}\},\{\mathrm{c}\},\{\mathrm{d}\},\{\mathrm{a}, \mathrm{b}\},\{\mathrm{c}, \mathrm{d}\}$

Here $\{b\}$ and $\{a, b\}$ are $g-m_{a}$ closed sets but not $g-m_{i}$ closed sets.

Remark 2.10. g- $\mathrm{m}_{\mathrm{a}}$ closed, closed sets $\mathrm{m}_{\mathrm{a}}$-closed sets and $\alpha$-closed sets are independent.

Example 2.11. Let $\mathrm{X}=\{\mathrm{a} \mathrm{b} \mathrm{c} d\}$ with $\tau=\{\mathrm{X}, \phi,\{\mathrm{a}\},\{\mathrm{a}, \mathrm{b}\},\{\mathrm{c}, \mathrm{d}\},\{\mathrm{a}, \mathrm{c}, \mathrm{d}\}\}$

Open sets $=\mathrm{X}, \phi,\{\mathrm{a}\},\{\mathrm{a}, \mathrm{b}\},\{\mathrm{c}, \mathrm{d}\},\{\mathrm{a}, \mathrm{c}, \mathrm{d}\}$

Closed sets $=\mathrm{X}, \phi,\{\mathrm{b}\},\{\mathrm{a}, \mathrm{b}\},\{\mathrm{c}, \mathrm{d}\},\{\mathrm{b}, \mathrm{c}, \mathrm{d}\}$

$\mathrm{m}_{\mathrm{a}}-$ closed $=\{\mathrm{a}, \mathrm{b}\},\{\mathrm{b}, \mathrm{c}, \mathrm{d}\}$

$\mathrm{g}-\mathrm{m}_{\mathrm{a}}$ closed $=\phi,\{\mathrm{b}\},\{\mathrm{c}\},\{\mathrm{d}\},\{\mathrm{a}, \mathrm{b}\},\{\mathrm{c}, \mathrm{d}\}$

$\mathrm{g}-\mathrm{m}_{\mathrm{i}}-$ open $=\mathrm{X},\{\mathrm{a}, \mathrm{b}\},\{\mathrm{c}, \mathrm{d}\},\{\mathrm{a}, \mathrm{b}, \mathrm{c}\},\{\mathrm{a}, \mathrm{b}, \mathrm{d}\},\{\mathrm{a}, \mathrm{c}, \mathrm{d}\}$.

$\alpha$-closed sets $=\phi,\{b\},\{a, b\},\{c, d\},\{b, c, d\}$

Remark 2.12. From the above implications we have the following as given below

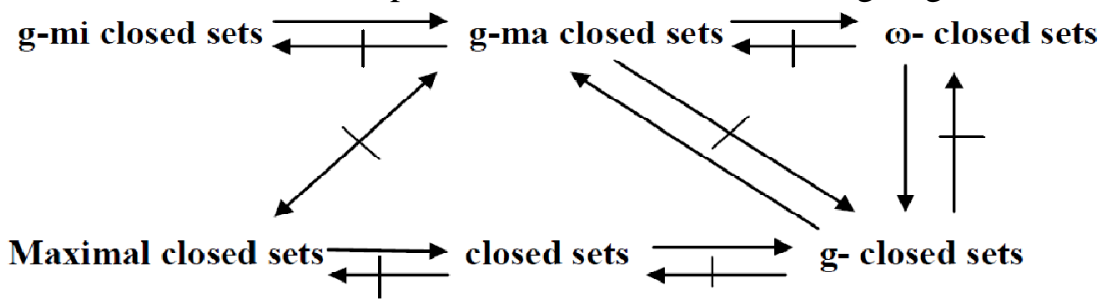

Figure 1:

Theorem 2.13. Intersection of any two $g-m_{a}$ closed is $g-m_{a}$ closed

Proof: Let A \& B be any two non-empty g- $\mathrm{m}_{\mathrm{a}}$ closed set. Then by definition 2.1

$\mathrm{cl}(\mathrm{A}) \subseteq \mathrm{U}$, whenever $\mathrm{A} \subseteq \mathrm{U}$ where $\mathrm{U}$ is $\mathrm{m}_{\mathrm{a}}-$ open, also $\mathrm{cl}(\mathrm{B}) \subseteq \mathrm{U}$, whenever $\mathrm{B} \subseteq \mathrm{U}$ where $\mathrm{U}$ is $\mathrm{m}_{\mathrm{a}}$-open. We know that $\mathrm{cl}(\mathrm{A} \cap \mathrm{B}) \subseteq \operatorname{cl}(\mathrm{A}) \cap \mathrm{cl}(\mathrm{B})$ also, since $\mathrm{cl}(\mathrm{A}) \subseteq \mathrm{U}$ whenever $\mathrm{A} \subseteq \mathrm{U}, \mathrm{U}$ is $\mathrm{m}_{\mathrm{a}}$-open\& $\mathrm{cl}(\mathrm{B}) \subseteq \mathrm{U}$, whenever $\mathrm{B} \subseteq \mathrm{U}, \mathrm{U}$ is $\mathrm{m}_{\mathrm{a}}-$ open.Thus $\operatorname{cl}(\mathrm{A} \cap \mathrm{B}) \subseteq \mathrm{U}$ whenever $\mathrm{A} \subseteq \mathrm{U} \& \mathrm{~B} \subseteq \mathrm{U}, \mathrm{U}$ is $\mathrm{m}_{\mathrm{a}}$-open. Therefore $\operatorname{cl}(\mathrm{A} \cap \mathrm{B}) \subseteq \mathrm{U}$ whenever $\mathrm{A} \cap \mathrm{B} \subseteq \mathrm{U}$ $\& \mathrm{U}$ is $\mathrm{m}_{\mathrm{a}}$-open. Therefore $\mathrm{A} \cap \mathrm{B}$ is $\mathrm{g}-\mathrm{m}_{\mathrm{a}}$ closed sets.

Remark 2.14. Union of any two $g-m_{a}$ closed sets may not be $g-m_{a}$ closed sets.

Example 2.15. From Example 2.4,clearly $\{b\} \&\{c\}$ are $g-m_{a}$ closed sets but $\{b c\}$ is not $\mathrm{g}-\mathrm{m}_{\mathrm{a}}$ closed sets. 
Suwarnlatha N. Banasode and Mandakini A.Desurkar

Theorem 2.16. If $A$ and $B$ are any two $g-m_{a}$ closed then $\operatorname{cl}(A \cup B)=\operatorname{cl}(A) \cup \operatorname{cl}(B)$ Proof: Since, $\mathrm{A} \subseteq \mathrm{A} \cup \mathrm{B}$ we have $\operatorname{cl}(\mathrm{A}) \subseteq \operatorname{cl}(\mathrm{A} \cup \mathrm{B})$ and since $\mathrm{B} \subseteq \mathrm{A} \cup \mathrm{B}$ we have $\mathrm{cl}(\mathrm{B}) \subseteq \operatorname{cl}(\mathrm{A} \cup \mathrm{B})$. Therefore $\mathrm{cl}(\mathrm{A}) \cup \mathrm{cl}(\mathrm{B}) \subseteq \operatorname{cl}(\mathrm{A} \cup \mathrm{B})$ since $\mathrm{cl}(\mathrm{A})$ and $\mathrm{cl}(\mathrm{B})$ are $\mathrm{g}-\mathrm{m}_{\mathrm{a}}$ closed sets. Therefore $\mathrm{A} \subseteq \mathrm{cl}(\mathrm{A})$ and $\mathrm{B} \subseteq \mathrm{cl}(\mathrm{B})$ this implies $\mathrm{A} \cup \mathrm{B} \subseteq \mathrm{cl}(\mathrm{A}) \cup \mathrm{cl}(\mathrm{B})$. Thus $\operatorname{cl}(A) \cup \operatorname{cl}(B)$ is the closed containing $A \cup B$. Since $\operatorname{cl}(A \cup B)$ is the smallest closed set containing $\mathrm{A} \cup \mathrm{B}$. Therefore $\operatorname{cl}(\mathrm{A} \cup \mathrm{B}) \subseteq \operatorname{cl}(\mathrm{A}) \cup \operatorname{cl}(\mathrm{B}) \mathrm{Hence} \operatorname{cl}(\mathrm{A} \cup \mathrm{B})=\operatorname{cl}(\mathrm{A}) \cup \operatorname{cl}(\mathrm{B})$

Theorem 2.17. If $\mathrm{A}$ is $\mathrm{g}-\mathrm{m}_{\mathrm{a}}$ closed in a top space $(\mathrm{X}, \tau)$ then $\mathrm{cl}(\mathrm{A})-\mathrm{A}$ contains no non empty minimal closed set.

Proof: Let $\mathrm{F}$ be any minimal closed subset of $\mathrm{cl}(\mathrm{A})-\mathrm{A}$. Then $\mathrm{F}^{\mathrm{c}}$ is a maximal open subset. Therefore $\mathrm{F} \subseteq \mathrm{cl}(\mathrm{A})-\mathrm{A}=\mathrm{cl}(\mathrm{A}) \cap \mathrm{A}^{\mathrm{c}}$

This implies $\mathrm{F} \subseteq \mathrm{cl}(\mathrm{A})$ and $\mathrm{F} \subseteq \mathrm{A}^{\mathrm{c}}$, since $\mathrm{cl}(\mathrm{A}) \cap \mathrm{A}^{\mathrm{c}} \subseteq \mathrm{cl}(\mathrm{A})$ and

$\operatorname{cl}(A) \cap A^{c} \subseteq A^{c}$. Therefore $F \subseteq A^{c}$ this implies $A \subseteq F^{c}$, where $F^{c}$ is maximal open set. Since $A$ is $g-m_{a}$ closed set, we have by the definition $c l(A) \subseteq F^{c}$ whenever $A \subset F^{c} \& F^{c}$ is maximal open. Since $\mathrm{cl}(\mathrm{A}) \subseteq \mathrm{F}^{\mathrm{c}}$ this implies $\mathrm{F} \subseteq[\mathrm{cl}(\mathrm{A})]^{\mathrm{c}}$. Therefore $\mathrm{F}=\phi$

Theorem 2.18. If $A$ is a $g-m_{a}$ closed $\& A \subseteq B \subseteq c l(A)$ then $B$ is a $g-m_{a}$ closed set in a topological space in $(\mathrm{X}, \tau)$.

Proof: Let $B$ be any set such that $B \subseteq U \& U$ is maximal open set.From the hypothesis $\mathrm{A} \subseteq \mathrm{B} \subseteq \mathrm{cl}(\mathrm{A})$.Since $\mathrm{A}$ is $\mathrm{g}-\mathrm{m}_{\mathrm{a}}$ closed, then by the definition 2.1 we have $\operatorname{cl}(\mathrm{A}) \subseteq \mathrm{U}$ whenever $A \subseteq U$ \& $U$ is maximal open set.Since $A \subseteq B \subseteq \operatorname{cl}(A) \& \operatorname{cl}(A) \subseteq U$ this implies $\mathrm{B} \subseteq \mathrm{cl}(\mathrm{A}) \operatorname{cl}(\mathrm{B}) \subseteq \operatorname{cl}(\mathrm{cl}(\mathrm{A}))=\mathrm{cl}(\mathrm{A})$ this implies $\mathrm{cl}(\mathrm{B}) \subseteq \mathrm{cl}(\mathrm{A}) \subseteq \mathrm{U}$. Therefore $\mathrm{cl}(\mathrm{B}) \subseteq \mathrm{U}$, whenever $\mathrm{B} \subseteq \mathrm{U} \& \mathrm{U}$ is maximal open set. Therefore is $\mathrm{g}-\mathrm{m}_{\mathrm{a}}$ closed set.

Theorem 2.19. If $\mathrm{A}$ is a $\mathrm{g}-\mathrm{m}_{\mathrm{a}}$ closed set in a topological space $(\mathrm{X}, \tau)$ then $\mathrm{cl}(\mathrm{A})-\mathrm{A}$ has no non empty minimal closed set.

Proof : Let $U$ be minimal closed subset of $\operatorname{cl}(A)-A$, then $U^{c}$ is maximal open set. $U \subseteq$ $\operatorname{cl}(\mathrm{A})$ - $\mathrm{A}$ this implies $\mathrm{U} \subseteq \operatorname{cl}(\mathrm{A}) \cap \mathrm{A}^{\mathrm{c}}$. Thus $\mathrm{U} \subseteq \operatorname{cl}(\mathrm{A})$ and $\mathrm{U} \subseteq \mathrm{A}^{\mathrm{c}}$ this implies $\mathrm{A} \subseteq \mathrm{U}^{\mathrm{c}}$ where $\mathrm{U}^{\mathrm{c}}$ is maximal open set. Since $A$ is $g-\mathrm{m}_{\mathrm{a}}$ closed set then by definition $2.1 \mathrm{cl}(\mathrm{A}) \subseteq$ $\mathrm{U}^{\mathrm{c}}$ which implies $\mathrm{U} \subseteq[\mathrm{cl}(\mathrm{A})]^{\mathrm{c}}$ also $\mathrm{U} \subseteq \mathrm{cl}(\mathrm{A})$. Therefore $\mathrm{U} \subseteq[\mathrm{cl}(\mathrm{A})]^{\mathrm{c}} \cap \operatorname{cl}(\mathrm{A})=\phi$. Therefore $\mathrm{U}=\phi$.

Theorem 2.20. If $\mathrm{A}$ is a $\mathrm{g}-\mathrm{m}_{\mathrm{a}}$ closed set in a topological space $(\mathrm{X}, \tau)$ then $\mathrm{cl}(\mathrm{A})-\mathrm{A}$ has no non emptyclosed subset.

Proof: The proof is omitted has it is obvious from the above Theorem 2.19.

Remark 2.21. If $A$ is the only maximal open set in a topological space then $g-m_{a}$ closed set is a null set.

Example 2.22. Let $\mathrm{X}=\{\mathrm{a}, \mathrm{bc}, \mathrm{d}\}$ and $\tau=\{\phi,\{\mathrm{a}\},\{\mathrm{b}\},\{\mathrm{a}, \mathrm{b}\},\{\mathrm{a}, \mathrm{b}, \mathrm{c}\}\}$.Maximal open set $=\{a, b, c\}$ therefore $g-m_{a}$ closed set $=\{\phi\}$.

Remark 2.23. If A is maximal open set and $\mathrm{g}-\mathrm{m}_{\mathrm{a}}$ closed set then $\mathrm{A}$ is a closed set. 
Generalized Maximal Closed Sets in Topological Space

Example 2.24. From example $2.11\{\mathrm{a}, \mathrm{b}\}$ is both g-ma closed set and maximal open set. Thus $\{a, b\}$ is a closed set in $X$.

\section{Generalized minimal open sets}

Definition 3.1. A subset $U$ of $X$ is said to be generalized minimal open sets iff its complement is generalized maximal closed set.

Remark 3.2. For any subset $A$ of $X, \operatorname{int}^{*}\left(c^{*}(A)-A\right)=\phi$

Remark 3.3. For any subset $A$ of $X, \operatorname{cl}^{*}(X-A)=X-\operatorname{int}^{*}(A)$

Theorem 3.4. Every $g-m_{i}$ open set is g-open set.

Proof: This follows from the definition 3.1 and theorem 2.5.

Remark 3.5. The converse of the above theorem is not true.

Example 3.6. Let $X=\{a, b, c\}$ and $\tau=\{X, \phi,\{a\},\{b, c\}\}$. Then the set $A=\{b\}$ is g-open set but not $\mathrm{g}-\mathrm{m}_{\mathrm{i}}$ open set.

Theorem 3.7. Every $g-m_{i}$ open set is $\omega$ - open set.

Proof: This follows from the definition 3.1 and Theorem 2.2.

Remark 3.8. The converse of the above Theorem is not true.

Example 3.9. From Example $3.6 \mathrm{~A}=\{\mathrm{b}\}$ is $\omega$ - open set but not $g-\mathrm{m}_{\mathrm{i}}$ open set.

Remark 3.10. From the above implications we have the following as given below

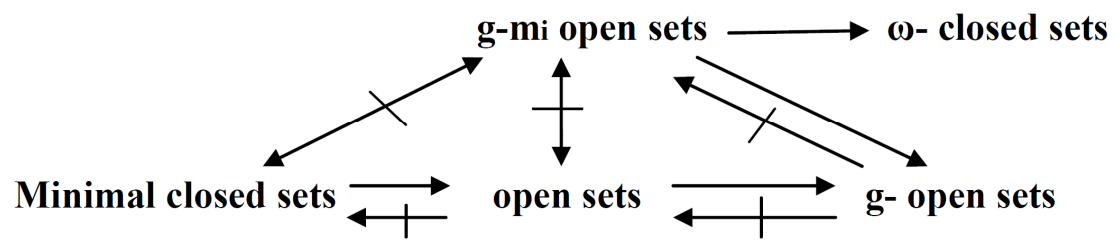

Figure 2:

Theorem 3.11. If $\mathrm{B}$ is $\mathrm{g}-\mathrm{m}_{\mathrm{i}}$ open iff $\mathrm{F} \subseteq \operatorname{int}(\mathrm{B})$ whenever $\mathrm{F} \subseteq \mathrm{B}$ and $\mathrm{F}$ is minimal closed set.

Proof: Let $\mathrm{B}$ be a $\mathrm{g}-\mathrm{m}_{\mathrm{i}}$ open set and $\mathrm{F}$ be a minimal closed set such that $\mathrm{F} \subseteq \mathrm{B}$ which implies $\mathrm{X}-\mathrm{B} \subseteq \mathrm{X}-\mathrm{F}$, where $\mathrm{X}-\mathrm{F}$ is maximal open set. Let $\mathrm{X}-\mathrm{B} \subseteq \mathrm{cl}^{*}(\mathrm{X}-\mathrm{B}) \subseteq \mathrm{X}-\mathrm{F}$ this implies $X$-int ${ }^{*}(\mathrm{~B}) \subseteq \mathrm{X}-\mathrm{F}$. Thus $\mathrm{F} \subseteq \operatorname{int}^{*}(\mathrm{~B}) \subseteq \operatorname{int}(\mathrm{B})$. Therefore $\mathrm{F} \subseteq$ int $(\mathrm{A})$.

Suppose $\mathrm{F}$ is minimal closed and $\mathrm{F} \subseteq$ int $\mathrm{B}$ whenever $\mathrm{F} \subseteq \mathrm{B}$. Let $\mathrm{X}-\mathrm{B} \subseteq \mathrm{U}$ where $\mathrm{U}$ is maximal open sets. Then $\mathrm{X}-\mathrm{U} \subseteq \mathrm{B}$ where $\mathrm{X}-\mathrm{U}$ is minimal closed set. Therefore by the hypothesis $X-U \subseteq \operatorname{int}(B)$ which implies $X-\operatorname{int}(B) \subseteq U$ which implies $\mathrm{cl}(\mathrm{X}-\mathrm{B}) \subseteq \mathrm{U}$ Therefore $\mathrm{X}-\mathrm{B}$ is generalized maximal closed set . Hence $\mathrm{B}$ is generalized minimal open set. 
Suwarnlatha N. Banasode and Mandakini A.Desurkar

Theorem 3.12. If int $A \subseteq B \subseteq A$ and $A$ is $g-m_{i}$ open then $B$ is $g-m_{i}$ open.

Proof: Let int $A \subseteq B \subseteq A$ and $A$ is $g-m_{i}$ open set. Then $A^{c} \subseteq B^{c} \subseteq(\operatorname{int}(A))^{c}$ this implies $\mathrm{A}^{\mathrm{c}} \subseteq \mathrm{B}^{\mathrm{c}} \subseteq \mathrm{cl}(\mathrm{A})^{\mathrm{c}}$. Since $\mathrm{A}^{\mathrm{c}}$ is $\mathrm{g}-\mathrm{m}_{\mathrm{a}}$ closed then by Theorem $2.18, \mathrm{~B}^{\mathrm{c}}$ isg- $\mathrm{m}_{\mathrm{a}}$ closed.Thus $\mathrm{B}$ is $g-m_{i}$ open set.

Theorem 3.13. If $A$ isg- $m_{i}$ open set in $X$ then $U=X$ whenever $U$ is an open set and $\operatorname{int}(\mathrm{A}) \cup \mathrm{A}^{\mathrm{c}} \subseteq \mathrm{U}$.

Proof: Let $A$ be $g-m_{i}$ open set in $X$. Let $U$ be an open set and int(A) $U A^{c} \subseteq U$ which implies $U^{c} \subseteq(\text { int } A)^{c} \cap A$. Since $A^{c}$ is $g-m_{a}$ closed set and $U^{c}$ is closed set it follows from Theorem 2.20 that $\mathrm{U}^{\mathrm{c}}=\phi$. Therefore $\mathrm{U}=\mathrm{X}$.

Remark 3.14. If $A$ is $g-m_{a}$ closed set then $\mathrm{cl}^{*}(A)$ - $A$ is $g-m_{i}$ open set.

\section{REFERENCES}

1. Levine, Norman, Generalized closed sets in topology, Rendicontidel Circolo Matematico di Palermo 19.1 (1970) 89-96.

2. S.S.Benchalli, S.N.Banasode and G.P.Siddapur, Generalized minimal closed sets in topological spaces, Journal of Computer and Mathematical Sciences, 1(6) (2010) 636-768.

3. F.Nakaoka and N.Oda, Some properties of maximal open sets, International Journal of Mathematics and Mathematical Sciences, 21 (2003) 1331-1340.

4. F.Nakaoka and N.Oda, Some applications of minimal open sets, International Journal of Mathematics and Mathematical Sciences 27(8) (2001) 471-476.

5. F.Nakaoka and N.Oda, On minimal closed sets, Proceedings of Topological Spaces Theory and its Applications, 5 (2003) $19-21$.

6. A.Mukharjee, On maximal, minimal open and closed sets, Commun. Korean Math. Soc, 30(3) (2015) 277-282.

7. S.N.Banasode and A.Mandakini Desurkar, Generalized minimal closed sets in bitopological spaces, Annals of Pure and Applied Mathematics, 14(2) (2017) 269276. 